\title{
Event Horizon Telescope Evidence for Alignment of the Black Hole in the Center of the Milky Way With the Inner Stellar Disk
}

\section{Citation}

Psaltis, Dimitrios, Ramesh Narayan, Vincent L. Fish, Avery E. Broderick, Abraham Loeb, and Sheperd S. Doeleman. 2014. "Event Horizon Telescope Evidence for Alignment of the Black Hole in the Center of the Milky Way With the Inner Stellar Disk." The Astrophysical Journal 798 (1) (December 15): 15. doi:10.1088/0004-637x/798/1/15.

\section{Published Version}

doi:10.1088/0004-637X/798/1/15

\section{Permanent link}

http://nrs.harvard.edu/urn-3:HUL.InstRepos:27801312

\section{Terms of Use}

This article was downloaded from Harvard University's DASH repository, and is made available under the terms and conditions applicable to Other Posted Material, as set forth at http:// nrs.harvard.edu/urn-3:HUL.InstRepos:dash.current.terms-of-use\#LAA

\section{Share Your Story}

The Harvard community has made this article openly available.

Please share how this access benefits you. Submit a story.

Accessibility 


\title{
EVENT HORIZON TELESCOPE EVIDENCE FOR ALIGNMENT OF THE BLACK HOLE IN THE CENTER OF THE MILKY WAY WITH THE INNER STELLAR DISK
}

\author{
Dimitrios Psaltis ${ }^{1}$, Ramesh Narayan ${ }^{2}$, Vincent L. Fish ${ }^{3}$, Avery E. Broderick ${ }^{4,5}$, \\ Abraham Loeb ${ }^{2}$, And ShePerd S. Doeleman ${ }^{2,3}$ \\ ${ }^{1}$ Astronomy Department, University of Arizona, 933 North Cherry Avenue, Tucson, AZ 85721, USA; dpsaltis@email.arizona.edu \\ ${ }^{2}$ Harvard-Smithsonian CfA, 60 Garden Street, Cambridge, MA 02138, USA; rnarayan@cfa.harvard.edu \\ ${ }^{3}$ Massachusetts Institute of Technology, Haystack Observatory, Route 40, Westford, MA 01886, USA \\ ${ }^{4}$ Perimeter Institute for Theoretical Physics, 31 Caroline Street North, Waterloo, ON, N2L 2Y5, Canada; abroderick@ perimeterinstitute.ca \\ ${ }^{5}$ Department of Physics and Astronomy, University of Waterloo, 200 University Avenue West, Waterloo, ON, N2L 3G1, Canada \\ Received 2014 April 29; accepted 2014 September 12; published 2014 December 15
}

\begin{abstract}
Observations of the black hole in the center of the Milky Way with the Event Horizon Telescope at $1.3 \mathrm{~mm}$ have revealed a size of the emitting region that is smaller than the size of the black-hole shadow. This can be reconciled with the spectral properties of the source, if the accretion flow is seen at a relatively high inclination $\left(50^{\circ}-60^{\circ}\right)$. Such an inclination makes the angular momentum of the flow, and perhaps of the black hole, nearly aligned with the angular momenta of the orbits of stars that lie within $\simeq 3^{\prime \prime}$ from the black hole. We discuss the implications of such an alignment for the properties of the black hole and of its accretion flow. We argue that future Event Horizon Telescope observations will not only refine the inclination of $\mathrm{Sgr} \mathrm{A}^{*}$ but also measure precisely its orientation on the plane of the sky.
\end{abstract}

Key words: Galaxy: center - relativistic processes - stars: black holes

\section{INTRODUCTION}

Observations of tight correlations between the properties of galaxies and of their central, supermassive black holes (e.g., Ferrarese \& Merritt 2000; Gebhardt et al. 2000) provide strong evidence that their formation and growth histories are tightly coupled (cf., e.g., Kormendy \& Ho 2013 for a recent review). This connection is further strengthened by the observed similarity between the histories of star formation and the evolution of quasars (see Madau et al. 1996; Boyle \& Terlevich 1998) as well as by the connection between starbursts and active galactic nucleus (AGN) activity (Kauffmann et al. 2003).

An important element in modeling the evolution of supermassive black holes is the orientation of their spins with respect to the planes of the galaxies in which they reside and to the angular momenta of the material that accretes on them (Dotti et al. 2013, and references therein). For example, black holes are believed to acquire most of their spins via accretion (Berti \& Volonteri 2008; Barausse 2012) and the magnitude of this effect depends on the relative alignment of the accretion flow with the black-hole spin. During mergers, black holes may receive substantial kicks depending on the relative orientation of the two merging objects (e.g., Baker et al. 2008). Finally, the interaction of the spin of a supermassive black hole with the accretion flow (Scheuer \& Feiler 1996; Martin et al. 2007; see also McKinney et al. 2013) or with the stars in its vicinity (Merritt \& Vasiliev 2012) leads to an alignment of the black hole with the inner regions of the galaxy and further affects the outcome of the above phenomena.

Observationally, the alignment of a black-hole spin with the axis of a galactic disk can be inferred primarily by indirect means. Statistical comparisons of the orientations of host galaxies with the AGN type of the black-hole accretion flows they harbor find some evidence for partial alignment (e.g., Lagos et al. 2011). On the other hand, individual comparisons of the relative orientations between galactic disks and radio jets (e.g., Schmitt et al. 2002) and between galactic disks and $\mathrm{H}_{2} \mathrm{O}$ maser sources (Greenhill et al. 2009) argue against such an alignment. The latter studies suggest that the accretion flows, which are responsible for launching the radio jets and for generating the masers, are not aligned with the disks of their hosts galaxies. However, due to obvious resolution limitations, such studies cannot address whether or not the inner accretion flows are aligned with the distribution of gas and stars in the vicinity of the black holes or with the black-hole spins themselves.

The Event Horizon Telescope (EHT) performs sub-millimeter very long baseline interferometry (VLBI) observations of the inner accretion flows around the black holes in the center of the Milky Way (Sgr A*; Doeleman et al. 2008) and of M87 (Doeleman et al. 2012). Because of its ability to resolve horizonscale structures, it provides unique probes of the inner accretion flows around these black holes, of the jet launching region in M87, and potentially of the orientations and magnitudes of the black-hole spins. In the case of $\operatorname{Sgr} \mathrm{A}^{*}$, the orientations of the stellar orbits within the central $0.1 \mathrm{pc}$ are also known (e.g., Bartko et al. 2009) and can be used in addressing directly the question of alignment between the inner stellar disk, the accretion flow, and the black-hole spin.

In this article, we argue that the measurement of the size of the emitting region of $\mathrm{Sgr} \mathrm{A}^{*}$ at $1.3 \mathrm{~mm}$ can be reconciled with the spectral properties of the source only if the latter is viewed at a relatively high inclination. Because of the small inferred size, the resulting inclination can be inferred robustly even with the current limited data and depends very weakly on the assumed thermodynamic properties of the accretion flow. Moreover, the inferred angular momentum of the inner accretion flow around Sgr A* is nearly aligned with that of the inner stellar disk, even though it is not aligned with the symmetry axis of the Milky Way. We conclude that either the black hole is not spinning rapidly or that the angular momentum of the black hole is also aligned with that of the stellar disk. 


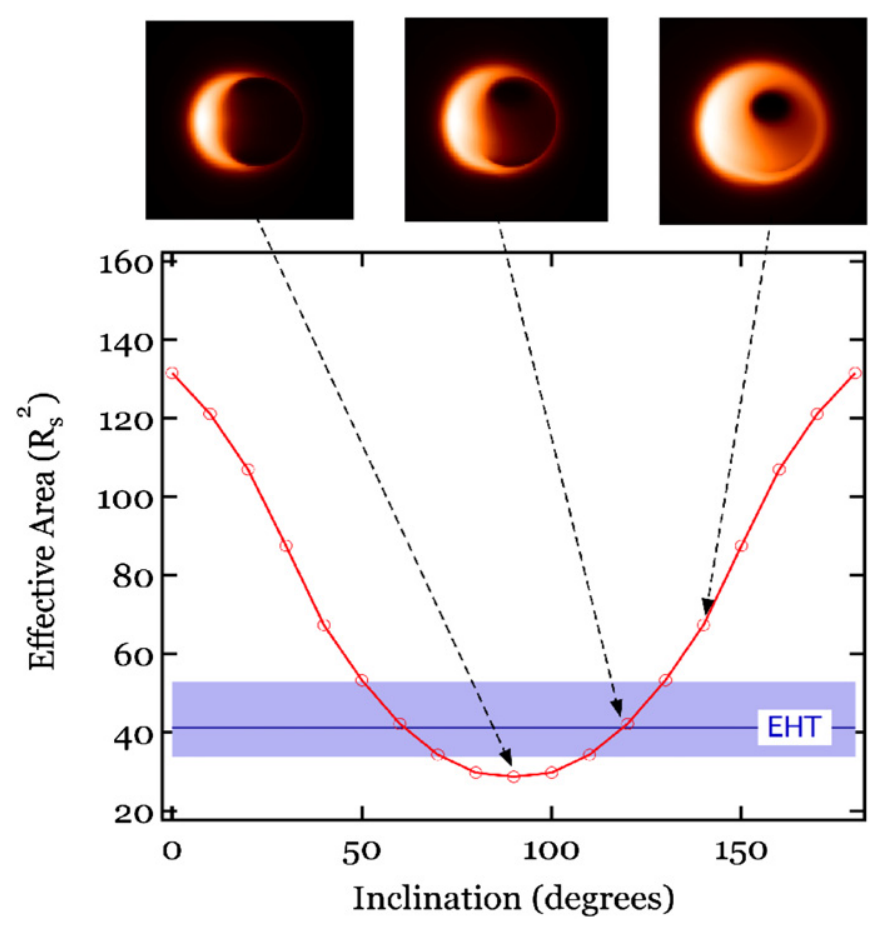

Figure 1. Dependence of the effective area of the image of a radiatively inefficient flow around $\mathrm{Sgr} \mathrm{A}^{*}$ on its inclination. All the images are calculated with accretion-flow parameters that reproduce, for each inclination, the observed submillimeter spectrum of the source. The horizontal line shows the measurement and the blue-shaded area the $99.7 \%$ uncertainty of effective area reported in 2008 by the EHT. Only for relatively high inclinations, at which Doppler effects boost the brightness of the approaching region of the flow and cause a large asymmetry in the image, can the observed flux from the source be reconciled with its relatively small size.

\section{THE ORIENTATION OF THE INNER ACCRETION FLOW AROUND Sgr A*}

Throughout this work, we define the orientation of an angular momentum vector in terms of two angles: the inclination $\theta$ measured with respect to the line of sight and the orientation $\phi$ of the projection of the vector in the sky measured in degrees north of east. This is the same coordinate system used by Bartko et al. (2009) in defining the orientations of the stellar orbits. The angle $\phi$ is related to the angle $\xi$ that is measured in degrees east of north (and that is often used in other articles of the sub-millimeter image of Sgr $\mathrm{A}^{*}$ ) via $\phi=90^{\circ}-\xi$.

\subsection{The Orientation Based on EHT Observations}

Observations of Sgr A* with the EHT have been reported for two epochs (2007 and 2009). In each case, the array resolved a source with a size of $\sim 40 \mu$ as, which translates to about four Schwarzschild radii. This size is consistently smaller than the expected diameter of the shadow of the black hole (Doeleman et al. 2008; Fish et al. 2011), which is about 5.2 Schwarzschild radii for zero spin.

The most straightforward way of understanding the fact that the emitting region in the accretion flow is smaller than the size of the shadow is to consider the effects of Doppler boosting on the emitted photons (Doeleman et al. 2008). Doppler effects increase the brightness of the approaching region of the accretion flow and reduce the brightness of the receding region. This leads to an asymmetric image with large brightness but very small effective area. In order for this interpretation to work,

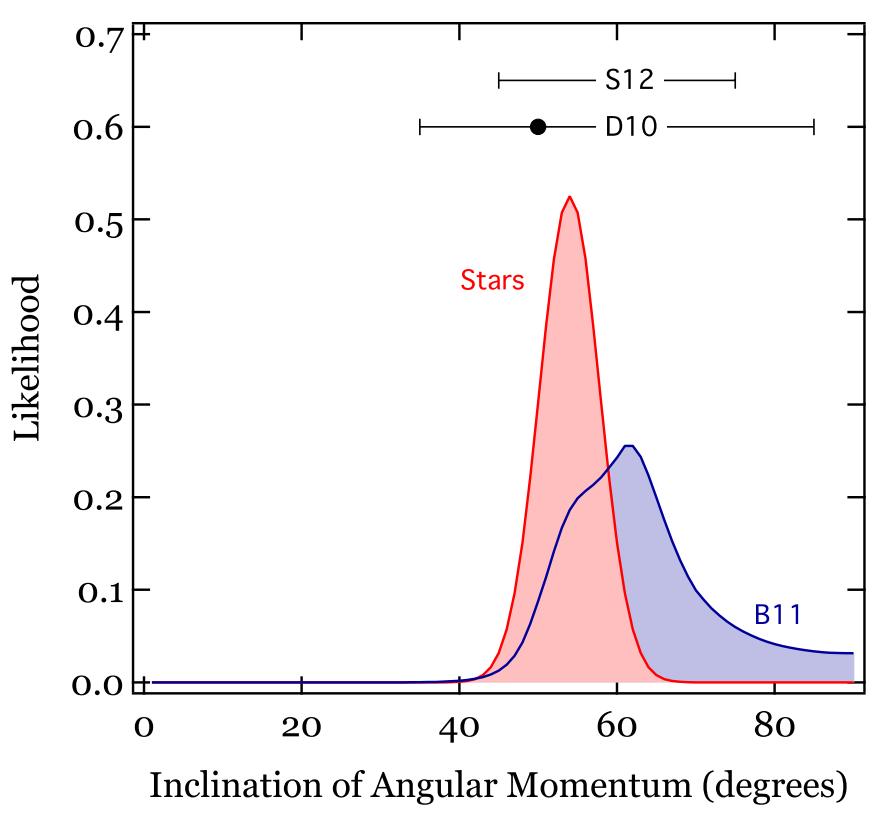

Figure 2. Posterior likelihood of (red) the inclination of the angular momentum of the stellar disk within 3".5 from Sgr A* (Bartko et al. 2009) and (blue) of the accretion flow, as inferred by Broderick et al. (2011a) when fitting analytical models of radiatively inefficient flows to observations made with the EHT. The horizontal error bars above the two curves show the most likely value and range for the inclination of the accretion flow, as inferred by Dexter et al. (2012) and by Shcherbakov et al. (2012), by fitting numerical GRMHD models to a subset of the same EHT observations.

however, the accretion flow needs to be observed at a relatively high inclination.

Figure 1 shows the dependence on inclination of the effective area of the accretion flow imaged at $1.3 \mathrm{~mm}$, such that at each inclination, the parameters of the accretion model acquire the value necessary to emit the observed flux. The model images used for this figure are from Broderick et al. (2011a) and the effective area was calculated for each inclination as the intensity weighted angular area of the image. The blue-shaded area shows the area of the image detected during the original observation with the EHT, assuming a circular Gaussian shape (Doeleman et al. 2008). The observed flux and the small size of the emitting region can be accommodated within this model only if the accretion flow is observed at a $\sim 60^{\circ}$ inclination.

Even though the above argument provides a good illustration of the way in which Doppler effects render the predicted $1.3 \mathrm{~mm}$ size of $\mathrm{Sgr} \mathrm{A}^{*}$ comparable to the observed one, it is not conclusive. Figure 1 compares haphazardly an intensity weighted area of the crescent-shaped predicted image to the effective area of a Gaussian that was assumed in fitting the observations. In fact, a uniform ring of emission, with a size consistent with the opening angle of the black-hole shadow was also used by Doeleman et al. (2009) to fit the initial EHT data. Subsequent observations of Sgr A* with the EHT allowed for a partial coverage of the $u-v$ plane, predominantly along E-W baselines. Broderick et al. (2011a) fit an analytical model of the accretion flow to the observed visibilities and obtained the posterior likelihood for the inclination of the angular momentum of the inner accretion flow shown in Figure 2. The most likely values for the inclination were found to be $\theta_{\mathrm{BH}}=68_{-20}^{+5} \mathrm{deg}$, where the errors correspond to a $68 \%$ confidence level. This is consistent with the value inferred in Figure 1 using the simpler argument based on the image size. (Note here that the posterior 
likelihood shown in Figure 2 peaks at a slightly different angle because of the marginalization over $\phi_{\mathrm{BH}}$.) Because of the up-down symmetry of the accretion model, there is a degeneracy with inclinations that are supplementary to those shown in Figure 2.

The measurement of the inclination depends rather weakly on the accretion model, as long as the flow remains nearly equatorial. The small size of the emitting region makes the result independent of the details of the accretion flow at larger scales. What we observe is primarily emission from a very localized region of the accretion flow that lies close to the innermost stable circular orbit and is pointing toward the Earth. Indeed, a similar analysis by Dexter et al. $(2010,2012)$, who used threedimensional GRMHD models of the accretion flow that are both time-dependent and highly turbulent, resulted in a very similar inference for the inclination angle: $\theta_{\mathrm{BH}}=60^{\circ} \pm 15^{\circ}$. In similar studies, Huang et al. $(2007,2009)$ estimated an inclination of $45^{\circ}$. Finally, Shcherbakov et al. (2012) obtained a good fit of their GRMHD models to the data for inclinations in the range of $45^{\circ}-75^{\circ}$, with the lowest inclinations being preferred if the black hole is spinning slowly.

Note here that the ranges of inclinations inferred in the above studies have been marginalized over the inferred orientations of the projection of the angular momentum vector on the sky. The latter quantity is not well determined by the current EHT observations, primarily because of the lack of substantial N-S baselines. Broderick et al. (2011a) give $\phi_{\mathrm{BH}}=142_{-17}^{+15} \mathrm{deg}$ and Dexter et al. (2012) give $\phi_{\mathrm{BH}}=160_{-86}^{+15}$. Because only the amplitudes (and not the phases) of the interferometric visibilities were used in these studies, there is a degeneracy between these values and values that are offset by $180^{\circ}$. Taking into account this degeneracy as well as the large measurement uncertainties leads to a very poor determination of the orientation of the angular momentum vector on the sky. In Section 4, we identify the optimal baselines at which a measurement of the correlated flux densities will allow for a precise inference of this angle. It is worth emphasizing, however, that the most likely values of the inclination depend rather weakly on the orientation of the angular momentum vector on the sky because the size of the image is determined primarily by Doppler effects, as discussed above.

The most significant assumption in the above argument is related to the alignment of the angular momentum of the accretion flow with the spin of the black hole. Dexter \& Fragile (2013) argued that if the black hole is spinning moderately to fast, its angular momentum vector is misaligned from the angular momentum vector of the accretion flow, and accretion proceeds via a geometrically thick flow, then the size of the millimeter image of Sgr A* will not provide a clear measure of its inclination. Instead, the shape of the image will be dominated by emission from the shocks between differentially precessing fluid elements. This will cause the apparent size of the emitting region to be small; as such, it will not provide a good measure of the Doppler boosts between the approaching and receding regions of the flow. Even though the current data are insufficient to distinguish between the two possibilities, the similarity in the visibility amplitudes between observations separated by several years (Broderick et al. 2011a) suggests that the millimeter image of $\mathrm{Sgr} \mathrm{A}^{*}$ is not dominated by transient emission, as we would expect from shocked material. Future EHT observations will have the sensitivity to clearly disentangle such time-variable structures, if they exist (Doeleman et al. 2009; Fish et al. 2009).

\subsection{Alignment of the Accretion Flow with the Stellar Disk}

The orientation and inclination of the inner accretion flow around Sgr A*, as inferred in the previous section, is misaligned with that of the galactic plane, for which $\theta_{\mathrm{MW}} \simeq 90^{\circ}$ and $\phi_{\mathrm{MW}} \simeq 330^{\circ}$ (Reid \& Brunthaler 2004). This is not surprising, however, because the stars within a few arcseconds from Sgr A*, for which monitoring of their orbits has been possible, appear to lie predominantly in a series of concentric disks that are inclined with respect to the galactic plane.

It is widely believed that the majority of stars orbiting the black hole at projected distances in the range $\simeq 1^{\prime \prime}-10^{\prime \prime}$ lie on a clockwise disk (Levin \& Beloborodov 2003; Genzel et al. 2003; Paumard et al. 2006; Lu et al. 2009; Bartko et al. 2009). There is evidence that the disk is warped (Bartko et al. 2009) with an orientation that evolves with distance. However, the orbital angular momentum vectors of the stars with projected distances that are closest to Sgr A* point, on average, toward a direction characterized by $\theta_{\mathrm{CW}}=54^{\circ} \pm 3.2$ and $\phi_{\mathrm{CW}}=$ $256^{\circ} \pm 3.2$ (Bartko et al. 2009), with a distribution that has a half-width at half-maximum (HWHM) of $\simeq 16^{\circ}$. A different, counterclockwise stellar disk at similar projected distances has also been suggested with an inclination of $\theta_{\mathrm{CCW}} \simeq 142^{\circ}$ and an orientation of $\phi_{\mathrm{CCW}} \simeq 200^{\circ}$ (Bartko et al. 2009; see also Genzel et al. 2003; Paumard et al. 2006; see, however, Lu et al. 2009; Yelda et al. 2014).

Recently, a cloud (G2) was discovered in the vicinity of Sgr A*(Gillessen et al. 2012), with a trajectory that eventually intercepted Sgr A* in mid-late 2013 (Gillessen et al. 2013). The orbital plane of the cloud was found to be within the HWHM of the orientations of the stellar orbits; Gillessen et al. (2012) give $\theta_{\mathrm{G} 2}=70.52$ and $\phi_{\mathrm{G} 2}=264.2$, whereas Phifer et al. (2013) infer $\theta_{\mathrm{G} 2}=59^{\circ} \pm 3^{\circ}$ and $\phi_{\mathrm{G} 2}=304^{\circ} \pm 11^{\circ}$.

Figure 2 compares the posterior likelihoods for the inclinations of the stellar disk to that of the inner accretion flow around Sgr A*, as inferred by EHT observations. This figure suggests that the angular momentum of the inner accretion flow may be aligned with the angular momenta of the stars in the inner disk.

Figure 3 shows the cumulative posterior likelihood that the inclinations of the two angular momentum vectors are within an angle $\Delta \theta_{0}$ by coincidence. In order to take into account the Lutz-Kelker bias (since the inclination angle can only be positive by definition), we generated a large number of Monte Carlo realizations of the two vectors with random directions in the sky. We then assigned Gaussian errors in the measurements of the inclinations of the two vectors, with a dispersion of $15^{\circ}$ for the black hole and of $3^{\circ}$ for the stellar disk, to match the observational errors. We finally calculated the fraction of the resulting measurements that lie within an angle $\Delta \theta_{0}$. The likelihood that the two inclinations are within $14^{\circ}$ by coincidence is $\lesssim 25 \%$. If we adopt the inclination inferred by Dexter et al. (2012) so that the two inclinations are within $6^{\circ}$ of each other, we find this likelihood to be $\lesssim 13 \%$.

\section{DISCUSSION}

In the previous section, we compared the inclination of the inner accretion flow around $\mathrm{Sgr} \mathrm{A}^{*}$, as inferred from observations with the EHT, to that of the stellar disk that lies within the central $3^{\prime \prime} .5$ and found them to be within $6^{\circ}-14^{\circ}$ of each other. We then used this evidence to argue that the angular momentum of the inner accretion flow around the black hole in the center of the Milky Way is nearly aligned with that of the stars close to it. 


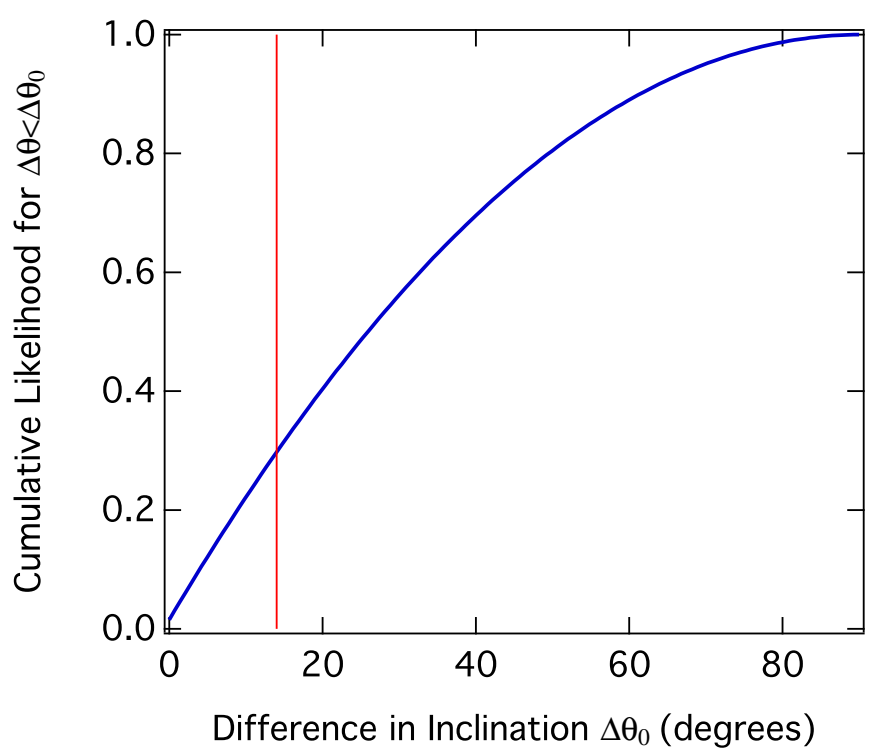

Figure 3. Posterior cumulative likelihood that the orientation of the inner accretion flow around Sgr $\mathrm{A}^{*}$ (as measured with the EHT) and of the inner stellar disk (as inferred from the stellar orbits) is within an angle $\Delta \theta_{0}$, if they are both randomly oriented in the sky. The two orientations align to within $14^{\circ}$ of each other by coincidence only $\lesssim 25 \%$ of the time.

It is worth pointing out that the inner accretion flow appears to be aligned with the stellar disk 3".5 away, even though a large number of stars (the S-star cluster) are closer to the black hole and have more isotropic orbits (see, e.g., Gillessen et al. 2009). This should not be very surprising, however, given that the stars at $1^{\prime \prime}-10^{\prime \prime}$ have much higher inferred wind mass-loss rates and are the ones that are probably supplying the accreting material to the black hole (see Figure 4 and Rockefeller et al. 2004; Cuadra et al. 2006).

The lack of variability in the size of the image of Sgr A* (see Fish et al. 2011; Broderick et al. 2011a) and the evidence shown in Figure 2 suggest that the emission from shocks due to the differential precession of nearby fluid elements is absent from the image of Sgr $\mathrm{A}^{*}$. This can be achieved either if the black-hole angular momentum is nearly aligned with that of the flow or if the black hole is slowly spinning (Dexter et al. 2012 suggest within $\simeq 15^{\circ}$ or for $a<0.3$ ).

The black hole may be aligned with the stellar cluster and the inner accretion flow either because of spin-orbit exchanges with the stellar cluster (Merritt \& Vasiliev 2012) or due to dissipation of its angular momentum in a geometrically thin disk (Scheuer $\&$ Feiler 1996). Merritt (2010) inferred that the inner stellar cluster has a mass of $\sim 10^{4} M_{\odot}$ and a core radius of $\sim 0.1 \mathrm{pc}$. Even though the orbital angular momentum in this cluster is comparable to that of the black hole, the mutual precession timescale is $>10^{10} \mathrm{yr}$ (Merritt \& Vasiliev 2012). Such a long timescale makes it highly unlikely that Sgr A* could have been aligned because of spin-orbit exchanges with the stellar cluster.

If Sgr A* was accreting at some point at a relatively high rate via a geometrically thin accretion disk, it would have aligned after a time of (Scheuer \& Feiler 1996; Volonteri et al. 2005)

$$
\begin{aligned}
t_{\text {align }} \simeq & 10^{7} \chi^{2 / 3}\left(\frac{\alpha}{0.1}\right)^{5 / 3}\left(\frac{\eta}{0.1}\right) \\
& \times\left(\frac{H}{0.1 R}\right)^{2 / 3}\left(\frac{\dot{M}}{0.1 \dot{M}_{\mathrm{Edd}}}\right)^{-1} \mathrm{yr},
\end{aligned}
$$

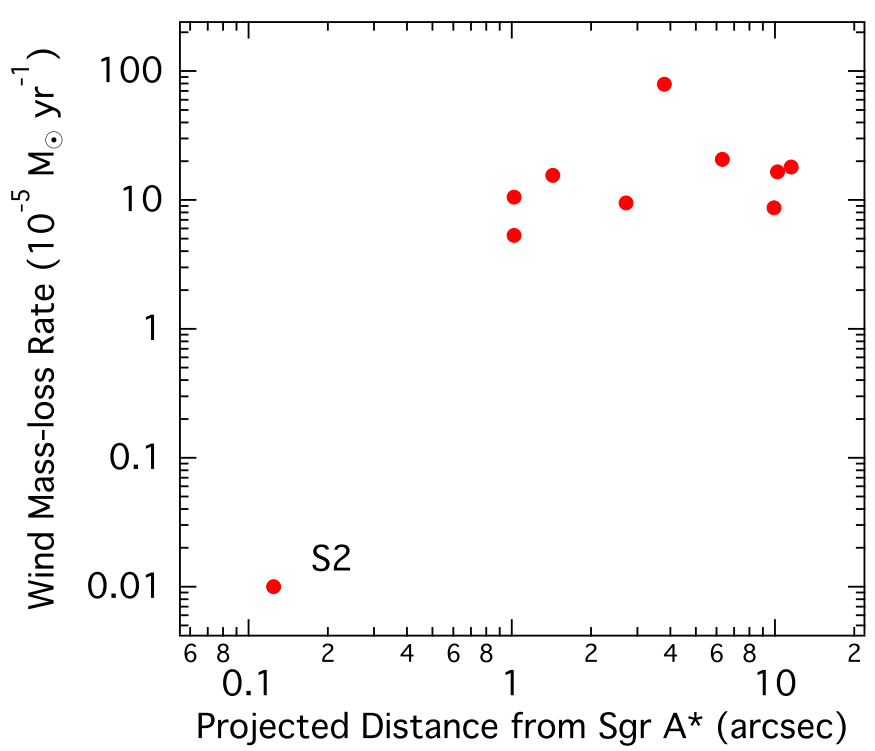

Figure 4. Inferred wind mass-loss rates for different stars in orbit around Sgr A*(from Najarro et al. 1997; Martins et al. 2007, 2008). The stars at angular distances $\simeq 1^{\prime \prime}-3^{\prime \prime}$, the orbits of which appear to be aligned with the inner accretion flow around the black hole, have the high mass-loss rates that are believed to be supplying the gas to the black hole.

where $\alpha$ is the viscosity parameter, $\chi$ is the spin of the black hole, $H$ is the scale height of the disk at distance $R$, and $\dot{M} / \dot{M}_{\mathrm{E}}$ is the mass accretion rate in units of the Eddington critical rate. In that amount of time, Sgr A* would have accreted a large fraction of its own mass and significantly more mass than is currently available within $0.1 \mathrm{pc}$, in the stellar cluster. This is, therefore, a highly unlikely possibility as well.

It is worth emphasizing here that relation (1) assumes that the only mechanism that causes the disk angular momentum to align with the black-hole spin is related to the Bardeen-Petterson effect (Bardeen \& Petterson 1975). If additional aligning mechanisms are effective, such as the one discussed in McKinney et al. (2013) that invokes large magnetic torques near spinning black holes with extensive jets, then the above timescale will be altered. However, such additional effects, which have comparable strength as the Bardeen-Petterson torques, will not significantly change the very long timescale calculated above. The most likely conclusion is that $\mathrm{Sgr} \mathrm{A}^{*}$ is slowly spinning, which is also indicated by the analysis of brightness distribution in the $1.3 \mathrm{~mm}$ images of the inner accretion flow (e.g., Broderick et al. 2011a).

Observations of the $1.3 \mathrm{~mm}$ image of $\mathrm{Sgr} \mathrm{A}^{*}$ with the EHT in the near future, when more stations are added to the interferometer, will break the degeneracies in the measurement of the two orientation angles and provide additional evidence to support or refute the result presented here.

First, in order for the array to maximize its ability to measure the orientation of the angular momentum of the accretion flow on the plane of the sky, a set of orthogonal baselines with a separation comparable to the projected size of the black-hole shadow must be used. This is demonstrated in Figure 5, which shows the scattering-broadened images and the corresponding $u-v$ maps for two different orientations of the accretion flow that are separated by $90^{\circ}$. For this figure, we have used the images of Broderick et al. (2011a) that best reproduce the currently observed spectra, polarization limits, and size of Sgr A*.

The black-hole shadow generates two null regions in the $u-v$ plane that are probed by a number of nearly orthogonal 

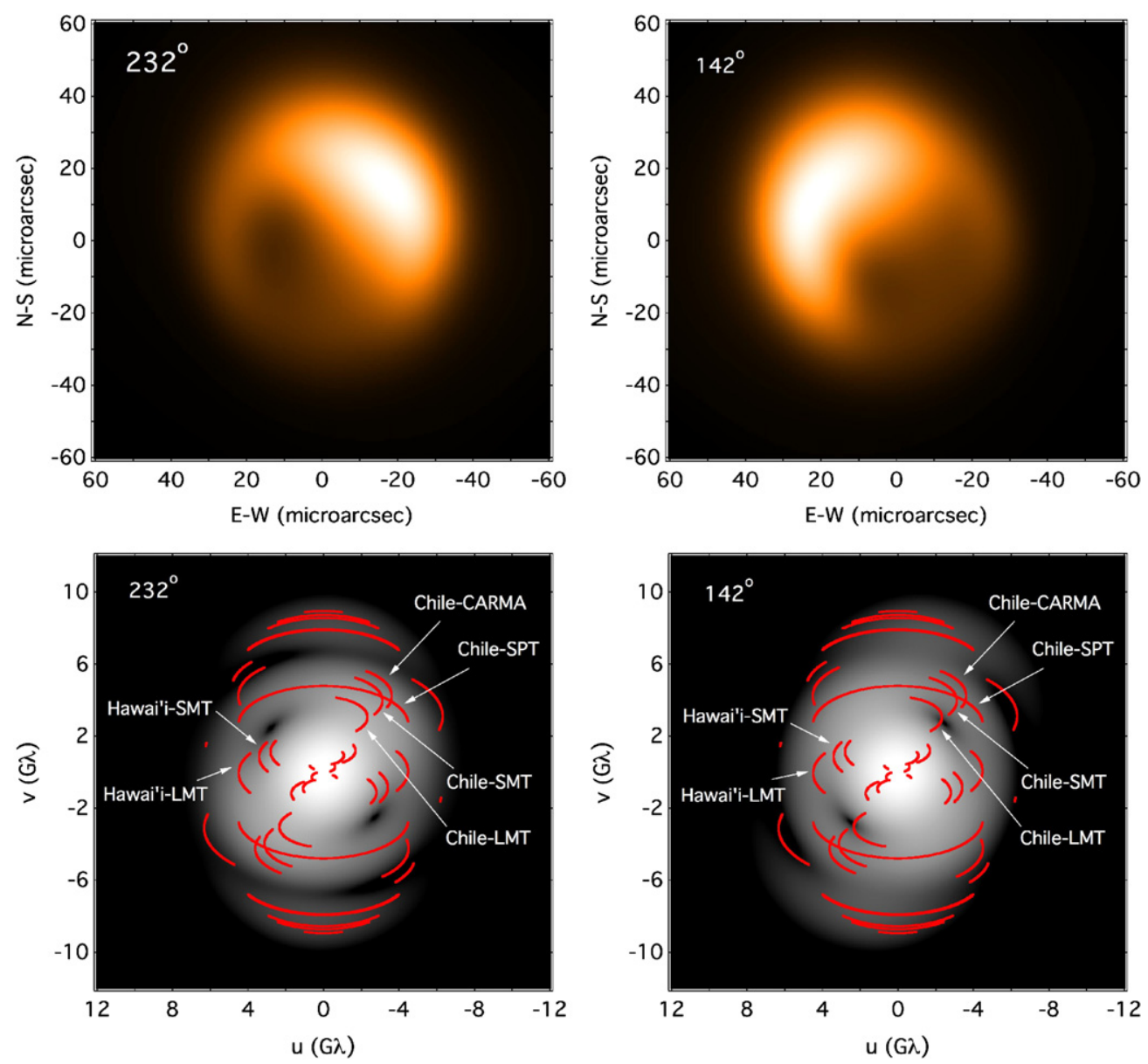

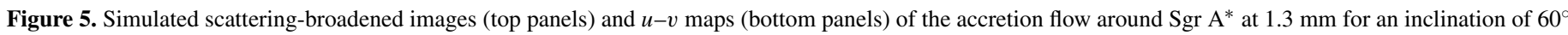

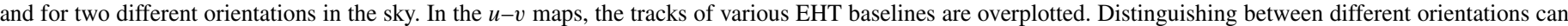

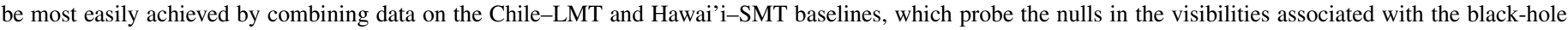
shadow.

baselines, e.g., the Chile-Large Millimeter Telescope (LMT) baseline in the $\mathrm{N}-\mathrm{S}$ direction and the Hawai' i-Submillimeter Telescope (SMT) baseline in the E-W direction. If the black-hole shadow is indeed imprinted on the image, then visibility amplitudes of these two baselines will be anticorrelated. Moreover, the ratio of the visibility amplitudes measured along these two baselines will provide a direct measure of the orientation of the angular momentum of the accretion flow in the sky (see Figure 6). In particular, if the inner accretion flow is aligned with the stellar disk (i.e., if $\phi=256^{\circ}$ ), then the two baselines should have comparable correlated flux densities that are of the order of $\sim 0.3 \mathrm{Jy}$.

When only the visibility amplitudes of the $u-v$ maps are used, then there is an additional degeneracy between orientations that differ by $180^{\circ}$. This degeneracy can be lifted with the use of closure phases that have already become available to $1.3 \mathrm{~mm}$ observations of Sgr A*(see Broderick et al. 2011b). Finally, if quasi-coherent regions of enhanced emission (i.e., blobs) are seen orbiting the black hole (see, e.g., Broderick \& Loeb 2006; Doeleman et al. 2009), then whether these bright regions appear to move clockwise or counterclockwise on the image plane will break the degeneracy between supplementary inclinations.

The orientation of the spin of Sgr A* can be also be inferred if a jet is discovered that originates from the central black hole. Several candidate jet structures have been identified during the last decade with orientations that practically cover the entire range of possibilities (see the discussion in Li et al. 2013). If the black-hole spin is aligned with the orbital angular momenta of the stars in the inner clockwise disk, which themselves are almost orthogonal to the orientation of the galactic pole, then the jet structures most recently identified by $\mathrm{Li}$ et al. (2013) and $\mathrm{Su}$ \& Finkbeiner (2012) cannot be related to Sgr A*. On the other hand, the structures identified by Mužić et al. (2007) and YusefZadeh et al. (2012) will be roughly aligned with the inferred spin axis of the central black hole. The situation is, of course, reversed if the orientation of the black-hole spin is at $\sim 142^{\circ}$, as inferred by Broderick et al. (2011a) using the limited EHT observations that are currently available. These considerations, albeit currently inconclusive, demonstrate how combining EHT observations with those of stellar orbits and jet-like structures in the galactic center can shed light to the complex interaction between the inner galaxy and its central black hole.

D.P. was supported by NSF CAREER award AST 0746549, NASA/NSF TCAN award NNX14AB48G, and by NSF award AST 1312034. R.N. was supported in part by NSF grant AST 1312651. A.L. was supported in part by NSF grants AST 0907890 and AST 1312034 and NASA grants NNX08AL43G and NNA09DB30A. Work at MIT Haystack Observatory was made possible by grants from the National Science Foundation (NSF) and the Gordon and Betty Moore Foundation (GBMF3561). A.E.B. receives financial support from the Perimeter Institute for Theoretical Physics and the Natural Sciences and Engineering Research Council of Canada through a Discovery 

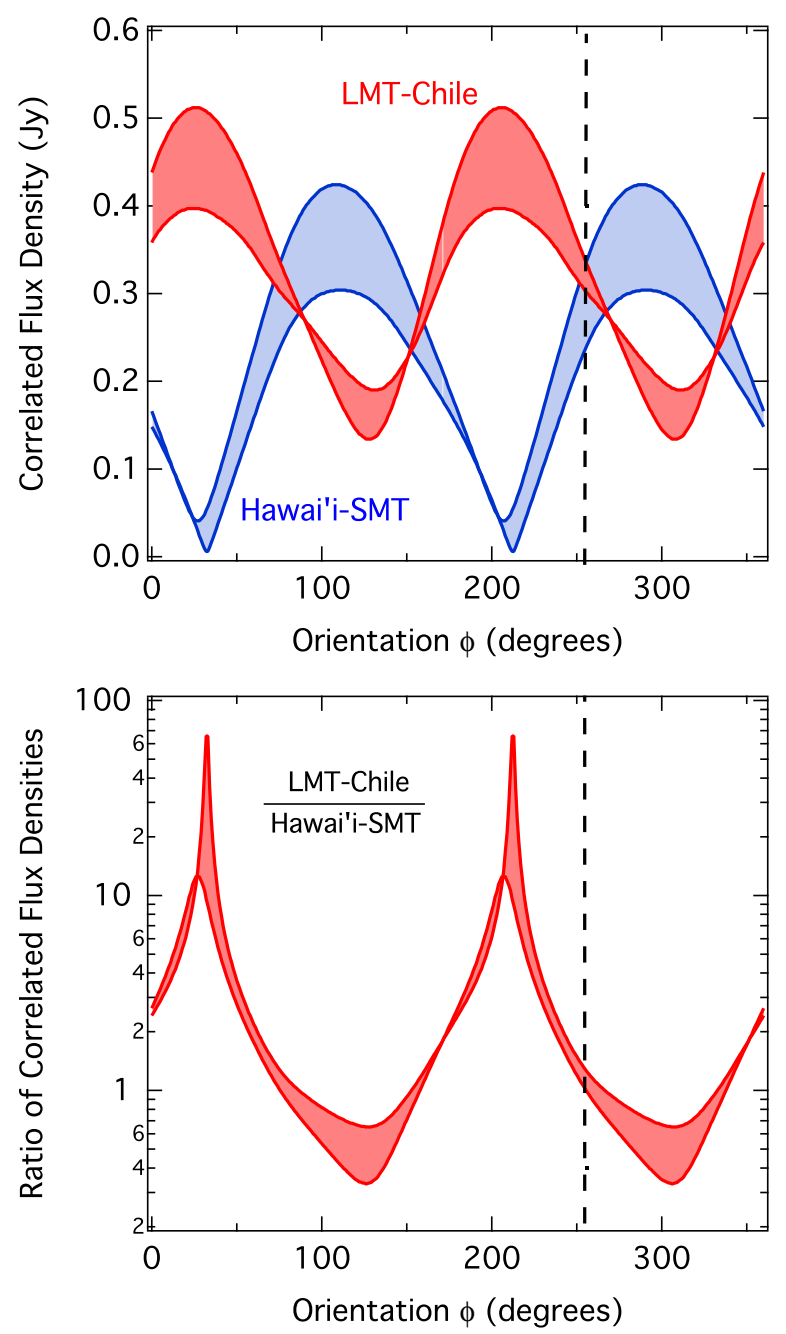

Figure 6. Predicted correlated flux densities for the LMT-Chile and Hawai'i-SMT baselines (top) and their ratios (bottom) as a function of the orientation of the inner accretion flow. The width of each curve shows the variation of the predicted flux densities with inclination in the $50^{\circ}-60^{\circ}$ range. The vertical dashed line shows the orientation of the inner stellar disk.

Grant. Research at Perimeter Institute is supported by the Government of Canada through Industry Canada and by the Province of Ontario through the Ministry of Research and Innovation.

\section{REFERENCES}

Baker, J. G., Boggs, W. D., Centrella, J., et al. 2008, ApJL, 682, L29 Barausse, E. 2012, MNRAS, 423, 2533
Bardeen, J. M., \& Petterson, J. A. 1975, ApJL, 195, L65

Bartko, H., Martins, F., Fritz, T. K., et al. 2009, ApJ, 697, 1741

Berti, E., \& Volonteri, M. 2008, ApJ, 684, 822

Boyle, B. J., \& Terlevich, R. J. 1998, MNRAS, 293, L49

Broderick, A. E., Fish, V. L., Doeleman, S. S., \& Loeb, A. 2011a, ApJ, 735, 110 Broderick, A. E., Fish, V. L., Doeleman, S. S., \& Loeb, A. 2011b, ApJ, 738, 38 Broderick, A. E., \& Loeb, A. 2006, MNRAS, 367, 905

Cuadra, J., Nayakshin, S., Springel, V., \& Di Matteo, T. 2006, MNRAS, 366,358

Dexter, J., Agol, E., Fragile, P. C., \& McKinney, J. C. 2010, ApJ, 717, 1092

Dexter, J., Agol, E., Fragile, P. C., \& McKinney, J. C. 2012, JPhCS, 372, 012023

Dexter, J., \& Fragile, P. C. 2013, MNRAS, 432, 2252

Doeleman, S. S., Fish, V. L., Broderick, A. E., Loeb, A., \& Rogers, A. E. E. 2009, ApJ, 695, 59

Doeleman, S. S., Fish, V. L., Schenck, D. E., et al. 2012, Sci, 338, 355

Doeleman, S. S., Weintroub, J., Rogers, A. E. E., et al. 2008, Natur, 455, 78

Dotti, M., Colpi, M., Pallini, S., Perego, A., \& Volonteri, M. 2013, ApJ, 762, 68

Ferrarese, L., \& Merritt, D. 2000, ApJL, 539, L9

Fish, V. L., Doeleman, S. S., Beaudoin, C., et al. 2011, ApJL, 727, L36

Fish, V. L., Doeleman, S. S., Broderick, A. E., Loeb, A., \& Rogers, A. E. E. 2009, ApJ, 706, 1353

Gebhardt, K., Bender, R., Bower, G., et al. 2000, ApJL, 539, L13

Genzel, R., Schödel, R., Ott, T., et al. 2003, ApJ, 594, 812

Gillessen, S., Eisenhauer, F., Trippe, S., et al. 2009, ApJ, 692, 1075

Gillessen, S., Genzel, R., Fritz, T. K., et al. 2012, Natur, 481, 51

Gillessen, S., Genzel, R., Fritz, T. K., et al. 2013, ApJ, 774, 44

Greenhill, L. J., Kondratko, P. T., Moran, J. M., \& Tilak, A. 2009, ApJ, 707, 787

Huang, L., Cai, M., Shen, Z.-Q., \& Yuan, F. 2007, MNRAS, 379, 833

Huang, L., Takahashi, R., \& Shen, Z.-Q. 2009, ApJ, 706, 960

Kauffmann, G., Heckman, T. M., Tremonti, C., et al. 2003, MNRAS, 346, 1055 Kormendy, J., \& Ho, L. C. 2013, ARA\&A, 51, 511

Lagos, C. D. P., Padilla, N. D., Strauss, M. A., Cora, S. A., \& Hao, L. 2011, MNRAS, 414, 2148

Levin, Y., \& Beloborodov, A. M. 2003, ApJL, 590, L33

Li, Z., Morris, M. R., \& Baganoff, F. K. 2013, ApJ, 779, 154

Lu, J. R., Ghez, A. M., Hornstein, S. D., et al. 2009, ApJ, 690, 1463

Madau, P., Ferguson, H. C., Dickinson, M. E., et al. 1996, MNRAS, 283, 1388

Martin, R. G., Pringle, J. E., \& Tout, C. A. 2007, MNRAS, 381, 1617

Martins, F., Genzel, R., Hillier, D. J., et al. 2007, A\&A, 468, 233

Martins, F., Gillessen, S., Eisenhauer, F., et al. 2008, ApJL, 672, L119

McKinney, J. C., Tchekhovskoy, A., \& Blandford, R. D. 2013, Sci, 339, 49

Merritt, D. 2010, ApJ, 718, 739

Merritt, D., \& Vasiliev, E. 2012, PhRvD, 86, 102002

Mužić, K., Eckart, A., Schödel, R., Meyer, L., \& Zensus, A. 2007, A\&A, 469, 993

Najarro, F., Krabbe, A., Genzel, R., et al. 1997, A\&A, 325, 700

Paumard, T., Genzel, R., Martins, F., et al. 2006, ApJ, 643, 1011

Phifer, K., Do, T., Meyer, L., et al. 2013, ApJL, 773, L13

Reid, M. J., \& Brunthaler, A. 2004, ApJ, 616, 872

Rockefeller, G., Fryer, C. L., Melia, F., \& Warren, M. S. 2004, ApJ, 604, 662

Scheuer, P. A. G., \& Feiler, R. 1996, MNRAS, 282, 291

Schmitt, H. R., Pringle, J. E., Clarke, C. J., \& Kinney, A. L. 2002, ApJ, 575,150

Shcherbakov, R. V., Penna, R. F., \& McKinney, J. C. 2012, ApJ, 755, 133

Su, M., \& Finkbeiner, D. P. 2012, ApJ, 753, 61

Volonteri, M., Madau, P., Quataert, E., \& Rees, M. J. 2005, ApJ, 620, 69

Yelda, S., Ghez, A. M., Lu, J. R., et al. 2014, ApJ, 783, 131

Yusef-Zadeh, F., Arendt, R., Bushouse, H., et al. 2012, ApJL, 758, L11 